\title{
African swine fever virus Georgia isolate harboring deletions of 9GL and MGF360/505 genes is highly attenuated in swine but does not confer protection against parental virus challenge
}

Vivian O’Donnell $^{1,3}$, Lauren G. Holinka ${ }^{1}$, Brenton Sanford ${ }^{2}$, Peter W. Krug ${ }^{1}$, Jolene Carlson ${ }^{1,5}$, Juan M. Pacheco ${ }^{2}$, Bo Reese ${ }^{4}$, Guillermo R. Risatti ${ }^{3}$, Douglas P. Gladue ${ }^{1}$ and Manuel V. Borca ${ }^{1 *}$

${ }^{1}$ Agricultural Research Service and ${ }^{2}$ Department of Homeland Security, Plum Island Animal Disease Center, Greenport, NY 11944, USA

${ }^{3}$ Department of Pathobiology and Veterinary Science and ${ }^{4}$ Center for Genome Innovation CAHNR, University of Connecticut, Storrs, CT 06269, USA

${ }^{5}$ Biosecurity Research Institute and Department of Diagnostic Medicine and Pathobiology, College of Veterinary Medicine, Kansas State University, Manhattan, KS 66506 


\begin{abstract}
African swine fever virus (ASFV) produces a contagious disease of domestic pigs that results in severe economic consequences to the swine industry. Control of the disease has been hampered by the unavailability of vaccines. We recently reported the development of two experimental vaccine strains (ASFV-G- $\triangle 9$ GL and ASFV-G- $\triangle$ MGF) based on the attenuation of the highly virulent and epidemiologically relevant Georgia2007 isolate. Deletion of the 9GL gene or six genes of the MGF360/505 group produced two attenuated ASFV strains which were able to confer protection to animals when challenged with the virulent parental virus. Both viruses, although efficient in inducing protection, present concerns regarding their safety. In an attempt to solve this problem we developed a novel virus strain, ASFV-G- $\triangle 9 \mathrm{GL} / \triangle \mathrm{MGF}$, based on the deletion of all genes deleted in ASFV-G$\Delta 9 \mathrm{GL}$ and ASFV-G- $\triangle \mathrm{MGF}$. ASFV-G- $\Delta 9 \mathrm{GL} / \Delta \mathrm{MGF}$ is the first derivative of a highly virulent ASFV field strain subjected to a double round of recombination events seeking to sequentially delete specific genes. ASFV-G- $\triangle 9 \mathrm{GL} / \Delta \mathrm{MGF}$ showed a decreased ability to replicate in primary swine macrophage cultures relative to that of ASFV-G and ASFV-G- $\triangle$ MGF but similar to that of ASFV-G$\Delta 9 \mathrm{GL} . \mathrm{ASFV}-\mathrm{G}-\Delta 9 \mathrm{GL} / \triangle \mathrm{MGF}$ was attenuated when intramuscularly inoculated into swine, even at doses as high as $10^{6} \mathrm{HAD}_{50}$. Animals infected with doses ranging from $10^{2}$ to $10^{6} \mathrm{HAD}_{50}$ did not present detectable levels of virus in blood at any time post-infection and they did not develop detectable levels of anti-ASFV antibodies. Importantly, ASFV-G- $\Delta 9$ GL/ $\triangle$ MGF does not induce protection against challenge with the virulent parental ASFV-G isolate. Results presented here suggest caution towards approaches involving genomic manipulations when developing rationally designed ASFV vaccine strains.
\end{abstract}




\section{Introduction}

African Swine Fever (ASF) is a highly lethal contagious viral disease of swine caused by ASF virus (ASFV), a large enveloped virus containing a double-stranded DNA genome of approximately 180-190 kilobase pairs (Costard et al., 2008). ASF causes a spectrum of disease, from highly lethal to sub-clinical, depending on host characteristics and the virus strain (Tulman et al., 2009). Virulent ASFV infections in domestic pigs are fatal and characterized by fever, hemorrhages, ataxia and severe depression.

Currently, ASF is endemic in several sub-Saharan African countries. In Europe, the disease is endemic in Sardinia (Italy) and outbreaks have been recorded in the Caucasus region since 2007, affecting Georgia, Armenia, Azerbaijan and Russia and more recently in Ukraine, Belarus, Lithuania, Latvia, Estonia and Poland, threatening to disseminate into neighbouring West European countries (Chapman et al., 2011).

There is no vaccine available for ASF and outbreaks are usually controlled by animal quarantine and elimination of affected animals. Experimental vaccines based on the use of different inactivated virus preparations have failed to induce protective immunity (Coggins, et al., 1974; Kihm et al., 1987; Mebus, C. A., 1988). Protective immunity does develop in pigs surviving viral infection against reinfection with homologous, although rarely to heterologous, viruses (Hamdy and Dardiri, 1984; Ruiz-Gonzalvo et al., 1986). Pigs immunized with live attenuated ASF viruses containing engineered individual deletions of ASFV virulence-associated genes (UK, DP69R, 23-NL, TK or 9GL genes) were protected when challenged with homologous virulent parental virus (Moore et al., 1998; Lewis et al., 2000; Zsak et al., 1996 and 1998). Thus far, these studies constitute the only experimental evidence describing the rational development of an effective live attenuated virus against ASFV. 
Following this rationale, we recently reported the development of two experimental vaccine strains (ASFV-G- $\Delta 9 \mathrm{GL}$ and ASFV-G- $\triangle \mathrm{MGF}$ ) based on the deletion of either the 9GL gene or six genes of MGF360/505 (O’Donnell et al., 2015a and 2015b). Both vaccine strains protected animals when challenged with the parental virus, the highly virulent Georgia2007 isolate. However, both viruses may present deficiencies regarding their safety. The ASFV-G- $\Delta 9 \mathrm{GL}$ protective dose is dangerously close to its lethal dose (O'Donnell et al., 2015b) while the genomic area modified in ASFV-G- $\triangle$ MGF is the target of active recombination events in ASFV (Krug et al., 2015; de la Vega et al., 1990; Pieres et al., 1997; Tabarez et al., 1987; Yanez et al., 1995), creating potential genetic instability. In order to develop a safer vaccine strain we decided to develop a novel virus where all genetic modifications present in ASFV-G- $\Delta 9 \mathrm{GL}$ and ASFV-G- $\Delta$ MGF were included in one strain, ASFV-G- $\Delta 9 \mathrm{GL} / \Delta \mathrm{MGF}$. ASFV-G- $\Delta 9 \mathrm{GL} / \Delta \mathrm{MGF}$ showed a decreased ability to replicate in primary swine macrophage cultures relative to that of ASFV-G and ASFV-G- $\triangle$ MGF and was drastically attenuated when intramuscularly inoculated in swine, even at doses as high as $10^{6} \mathrm{HAD}_{50}$. Animals infected with doses ranging from $10^{2}$ to $10^{6} \mathrm{HAD}_{50}$ presented no detectable viremias or anti-ASFV antibodies. Accordingly, ASFV-G- $\Delta 9 \mathrm{GL} / \Delta \mathrm{MGF}$ does not induce protection against challenge with virulent parental ASFV-G. Results presented here suggest caution towards approaches involving multiple genomic manipulations when developing rationally designed ASFV vaccine strains. 


\section{Materials and Methods}

\section{Cell cultures and viruses}

Primary swine macrophage cell cultures were prepared from defibrinated swine blood as previously described by Zsak et al. (1996). Briefly, heparin-treated swine blood was incubated at $37^{\circ} \mathrm{C}$ for 1 hour to allow sedimentation of the erythrocyte fraction. Mononuclear leukocytes were separated by flotation over a Ficoll-Paque (Pharmacia, Piscataway, N.J.) density gradient (specific gravity, 1.079). The monocyte/macrophage cell fraction was cultured in plastic Primaria (Falcon; Becton Dickinson Labware, Franklin Lakes, N.J.) tissue culture flasks containing macrophage media, composed of RPMI 1640 Medium (Life Technologies, Grand Island, NY) with 30\% L929 supernatant and 20\% fetal bovine serum (HI-FBS, Thermo Scientific, Waltham, MA) for 48 hours at $37^{\circ} \mathrm{C}$ under $5 \% \mathrm{CO}_{2}$. Adherent cells were detached from the plastic by using $10 \mathrm{mM}$ EDTA in phosphate buffered saline (PBS) and were then reseeded into Primaria T25, 6- or 96-well dishes at a density of $5 \times 10^{6}$ cells per $\mathrm{ml}$ for use in assays 24 hours later.

ASFV Georgia (ASFV-G) was a field isolate kindly provided by Dr. Nino Vepkhvadze, from the Laboratory of the Ministry of Agriculture (LMA) in Tbilisi, Republic of Georgia (Krug et al., 2015). ASFV-G- 49 GL has been previously developed and reported (O'Donnell et al., 2015b). Basically, ASFV-G- $\triangle 9$ GL was developed by deleting the $9 \mathrm{GL}$ gene from ASFV-G by homologous recombination, replacing it with the $\beta$-glucuronidase ( $\beta$-GUS) gene with the ASFV p72 late gene promoter (O’Donnell et al., 2015b).

Comparative growth curves between ASFV-G, ASFV-G- $\triangle 9$ GL, ASFV-G- $\triangle$ MGF and ASFVG- $\triangle 9 \mathrm{GL} / \triangle \mathrm{MGF}$ viruses were performed in primary swine macrophage cell cultures. Preformed monolayers were prepared in 24-well plates and infected at a MOI of 0.01 (based on $\mathrm{HAD}_{50}$ previously determined in primary swine macrophage cell cultures). After 1 hour of adsorption at 
$37^{\circ} \mathrm{C}$ under $5 \% \mathrm{CO}_{2}$ the inoculum was removed and the cells were rinsed two times with PBS. The monolayers were then rinsed with macrophage media and incubated for 2, 24, 48, 72 and 96 hours at $37^{\circ} \mathrm{C}$ under $5 \% \mathrm{CO}_{2}$. At appropriate times post-infection, the cells were frozen at $\leq-70^{\circ} \mathrm{C}$ and the thawed lysates were used to determine titers by $\mathrm{HAD}_{50} / \mathrm{ml}$ in primary swine macrophage cell cultures. All samples were run simultaneously to avoid inter-assay variability.

Virus titration was performed on primary swine macrophage cell cultures in 96-well plates. Virus dilutions and cultures were performed using macrophage medium. Presence of virus was assessed by hemadsorption (HA) and virus titers were calculated by the Reed and Muench method (1938).

\section{Construction of the recombinant ASFV-G- $\Delta$ 9GL/ $\Delta$ MGF}

Recombinant ASFV-G- $\Delta 9 \mathrm{GL} / \triangle \mathrm{MGF}$ was generated by homologous recombination between the parental ASFV-G- $\triangle 9$ GL genome and a recombination transfer vector following infection and transfection of swine macrophage cell cultures (Zsak et al., 1996). The recombinant transfer vector (p72GFP $\triangle \mathrm{MGF}$ ) contained flanking genomic regions, which included the amino terminus of the MGF505 1R gene mapping to the left (left arm is located between genomic positions 24566-27928) and the carboxyl end of the MGF505 3R gene to the right (right arm is located between genomic positions 35487-36515) and a reporter gene cassette containing the green fluorescent protein (GFP) gene with the ASFV p72 late gene promoter (Zsak et al., 1996). This construction created a 7558nucleotide deletion in the left variable region of ASFV-G (between nucleotide positions 27928 to 35481) (Fig. 1). Recombinant transfer vector p72GFP $\triangle$ MGF was obtained by DNA synthesis (Epoch Life Sciences, Sugar Land, TX, USA). Macrophage cell cultures were infected with ASFV-G and transfected with p72mGFP $\triangle \mathrm{MGF}$. Recombinant viruses representing independent primary foci were purified to homogeneity by successive rounds of limiting dilution purification. 


\section{Polymerase chain reaction $(\mathbf{P C R})$}

Purity of ASFV-G- $\Delta 9$ GL/ $\Delta$ MGF in the virus stock was assessed by PCR. Detection of the MGF350/MGF505 genes (as presence of parental genome DNA) was performed using the following pair of primers: forward 5'GAGGATGATTTGCCCTTCACTCA3'; reverse 5'CGCCACTAGTAAA CATTGTTCTATCT3'. These primers amplified a $422 \mathrm{bp}$ fragment of the ORF MGF505-1R gene. Detection of the $\beta$-Gus gene (as control of DNA availability), amplifying a 471 bp fragment, was performed using the following pair of primers: forward 5' GCCGATTATCATCACCGAATAC3'; reverse 5'TGCGCCAGGAGAGTTG TTGATTC3'.

\section{Next Generation Sequencing (NGS) of ASFV genomes}

ASFV DNA was extracted from infected cells and quantified as described earlier (Krug et al., 2015). Full-length sequencing of the virus genome was performed as described elsewhere (Krug et al., 2015). Briefly, one microgram of virus DNA was enzymatically sheared and the resulting fragmented DNA size distribution was assessed. Adapters and library barcodes were ligated to the fragmented DNA. The appropriate size range of the adapter-ligated library was collected using the Pippin Prep ${ }^{\mathrm{TM}}$ system (Sage Science) followed by normalization of library concentration. The DNA library was then clonally amplified onto ISPs and enriched. Enriched template ISPs were prepared and loaded onto Ion chips for sequencing using an Ion Torrent PGM ${ }^{\mathrm{TM}}$ instrument. Sequence analysis was performed using Galaxy (https://usegalaxy.org/) and CLC Genomics Workbench (CLCBio).

\section{Animal experiments}

Animal experiments were performed under biosafety level 3 conditions in the animal facilities at PIADC following a protocol approved by the Institutional Animal Care and Use Committee.

ASFV-G- $\Delta 9 \mathrm{GL} / \Delta \mathrm{MGF}$ was assessed for its virulence phenotype relative to the parental ASFV-G virus using 80-90 pound commercial breed swine. Five pigs were inoculated 
intramuscularly (IM) either with $10^{2}, 10^{4}$ or $10^{6} \mathrm{HAD}_{50}$ of ASFV-G- $\triangle 9 \mathrm{GL} / \Delta \mathrm{MGF}$ or $10^{4} \mathrm{HAD}_{50}$ of ASFV-G. Clinical signs (anorexia, depression, fever, purple skin discoloration, staggering gait, diarrhea and cough) and changes in body temperature were recorded daily throughout the experiment.

To assess the protective effect of the different doses of ASFV-G- $\Delta 9 \mathrm{GL} / \Delta \mathrm{MGF}$ at 28 days post-infection, ASFV-G- $\Delta 9 \mathrm{GL} / \Delta \mathrm{MGF}-$ infected animals were IM challenged with $10^{3} \mathrm{HAD}_{50}$ of highly virulent parental ASFV-G. Clinical signs (as described above) and changes in body temperature were recorded daily throughout the experiment.

\section{Detection of anti-ASFV antibodies}

Anti-ASFV antibodies in sera of infected animals were quantified using an in-house developed ELISA. Antigen preparation and ELISA procedure was based on the method of Katz et al., (2012) with minor adjustments. Briefly, Vero cells were infected with an ASFV-G strain adapted to replicate in Vero cells (Krug et al., 2015) until cytopathic effect reached 100\%. The infected cells were resuspended in water containing protease inhibitor (Roche, New York, NY), followed by the addition of Tween 80 (G-Biosciences, Saint Louis, MO) and sodium deoxycholate (Sigma, Saint Louis, MO) to a final concentration of $1 \%(\mathrm{v} / \mathrm{v})$. Uninfected Vero cells were treated in the same manner and these antigens were stored at $-70^{\circ} \mathrm{C}$. Maxisorb ELISA plates (Nunc, Saint Louis, MO) were coated with $1 \mu \mathrm{g}$ per well of either infected cell or uninfected cell antigen. The plates were blocked with phosphate buffered saline containing 10\% skim milk (Merck, Kenilworth, NJ) and 5\% normal goat serum (Sigma). Each swine serum was tested at multiple dilutions against both infected and uninfected cell antigen. ASFV-specific antibodies in the swine sera were detected by an antiswine IgG-horseradish peroxidase conjugate (KPL, Gaithersburg, MD) and SureBlue Reserve peroxidase substrate (KPL). Plates were read at OD630 in an ELx808 plate reader (BioTek, 
Shoreline, WA). Swine sera were considered positive for ASFV-specific antibodies if the OD630 ratio of the reaction against infected cell antigen to uninfected cell antigen was higher than 2.2. 


\section{Results and Discussion}

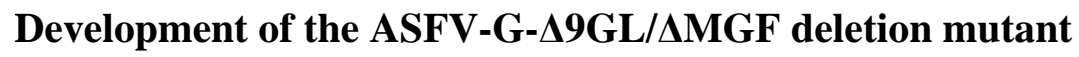

We hypothesized that a recombinant virus, ASFV-G- $\triangle 9 \mathrm{GL} / \Delta \mathrm{MGF}$, harboring all gene deletions individually present in $\mathrm{ASFV}-\mathrm{G}-\Delta 9 \mathrm{GL}$ and $\mathrm{ASFV}-\mathrm{G}-\Delta \mathrm{MGF}$ would potentially present a more attenuated phenotype. ASFV-G- $\Delta 9 \mathrm{GL} / \Delta \mathrm{MGF}$ was generated by homologous recombination between the recombinant ASFV-G- $\triangle 9$ GL, previously developed by O'Donnell et al., (2015b), and recombination transfer vector $\mathrm{p} 72 \mathrm{mGFP} \triangle \mathrm{MGF}$ by infection and transfection procedures using swine macrophage cell cultures (Zsak et al., 1996; O’Donnell et al., 2015a and 2015b). ASFV-G- $\Delta$ GGL has replaced its native 9GL gene with the ASFV p72 late gene promoter/ $\beta$-glucuronidase (GUS) gene cassette (O'Donnell et al., 2015b). Therefore, ASFV-G- $\triangle 9$ GL was used as parental virus in infection/transfection procedures performed on macrophage cell cultures using recombinant transfer vector p72mGFP $\triangle \mathrm{MGF}$ as previously described (see Material and Methods). The resulting recombinant virus, besides the deletion of the 9GL gene, harbors a deletion encompassing the carboxyl terminal half of the MGF505-1R gene, the complete deletion of MGF360-12L, 13L, 14L, and MGF505-2R genes along with the deletion of the amino terminal half of the MGF505-3R gene (O’Donnell et al., 2015a) (Fig. 1). The recombinant virus was obtained after eight successive limiting dilution purification events on primary swine macrophage cell cultures using GFP as the fluorescent selectable marker. The virus population obtained from the last round of purification was amplified in primary swine macrophage cell cultures to obtain a virus stock.

To ensure the absence of parental ASFV-G- $\Delta 9 \mathrm{G}$ in the ASFV-G- $\Delta 9 \mathrm{GL} / \Delta \mathrm{MGF}$ stock preparation, virus DNA was extracted from the virus stock and analyzed by PCR using primers targeting genes MGF and $\beta$-Gus. While amplicons for $\beta$-Gus genes were detected in DNA extracted 
from the virus stock, no amplicons were generated with primers targeting the MGF505-1R gene (Fig. 2 ), indicating the lack of contamination of the ASFV-G- $\Delta 9 \mathrm{GL} / \Delta \mathrm{MGF}$ stock with parental ASFV-G$\Delta 9 \mathrm{GL}$.

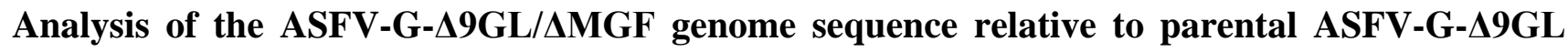 genome sequence}

To evaluate the accuracy of the genetic modification and the integrity of the genome of the newly developed recombinant virus, full genome sequences of ASFV-G- $\Delta 9 \mathrm{GL} / \Delta \mathrm{MGF}$ and parental ASFV-G- $\Delta 9$ GL were obtained using NGS on the Ion Torrent PGM ${ }^{\mathrm{TM}}$ and compared (Krug et al, 2014). The full-length genome comparison between ASFV-G- $\Delta 9 \mathrm{GL} / \Delta \mathrm{MGF}$ and parental ASFV-G$\Delta 9 \mathrm{GL}$ revealed a deletion of 7558 nucleotides corresponding with the introduced modification in the MGF360/505 area (Fig. 1). Additionally, the consensus sequence of the ASFV-G- $\Delta 9$ GL/ $\Delta$ MGF genome showed an insertion of 1256 nucleotides corresponding to the p72mGFP cassette sequence (Fig. 1). Besides the insertion of the p72mGFP $\triangle \mathrm{MGF}$ cassette, no additional differences were observed between the ASFV-G- $\Delta 9 \mathrm{GL} / \Delta \mathrm{MGF}$ and parental ASFV-G- $\Delta 9 \mathrm{GL}$ genomes, confirming ASFV-G- $\Delta 9 \mathrm{GL} / \Delta \mathrm{MGF}$ did not accumulate undesired mutations during the process of homologous recombination and plaque purification. It is interesting to note that successive passages of ASFV in swine macrophages cell cultures is often accompanied by genome modifications as we previously observed with several recombinant viruses produced under a process that included in some cases more than 15 passages.

\section{Replication of ASFV-G- 49 GL/AMGF in primary swine macrophages}

While viruses lacking the 9GL gene have been described as having a decreased ability to replicate in swine macrophages, viruses lacking the MGF genes located in the left variable region of the ASFV genome are still able to replicate in swine macrophages (Zack et al., 2001; Krug et al., 
2015; O'Donnell et al., 2015b). Therefore, we analyzed the in vitro growth characteristics of ASFVG- $\Delta 9 \mathrm{GL} / \Delta \mathrm{MGF}$ in swine macrophages, the primary cell targeted by ASFV during infection in swine, in comparison to those of ASFV-G- $\Delta 9 \mathrm{GL}$, ASFV-G- $\triangle$ MGF and field isolate ASFV-G (Krug et al., 2015; O'Donnell et al., 2015a and 2015b). Macrophage cell cultures were infected with one of these viruses at a MOI of 0.01 and samples were collected at 2, 24, 48, 72 and 96 hours post-infection (hpi). As expected, ASFV-G- $\triangle$ MGF presented growth kinetics indistinguishable from ASFV-G (O'Donnell et al., 2015a); however, both ASFV-G- $\Delta 9$ GL/ $\Delta$ MGF and ASFV-G- $\Delta 9$ GL displayed slower kinetics. Although all four viruses reached similar titers by the end of the experiment, ASFVG- $\Delta 9 \mathrm{GL} / \Delta \mathrm{MGF}$ and ASFV-G- $\Delta 9 \mathrm{GL}$ yields were 10 to 100 times lower than those of ASFV-G$\triangle \mathrm{MGF}$ and ASFV-G virus (Fig. 3) at some of the intermediate sampling time points. Therefore, as it happens with ASFV-G- $\mathrm{MGF}$ (O'Donnell et al., 2015a), deletion of these particular six MGF360/MGF505 genes does not significantly affect the ability of the mutated virus to replicate in primary swine macrophage cultures when compared with its corresponding parental virus (ASFV-G for ASFV-G- $\Delta$ MGF and ASFV-G- $\Delta 9 \mathrm{GL}$ for ASFV-G- $\Delta 9 \mathrm{GL} / \Delta \mathrm{MGF}$ ).

\section{Assessment of ASFV-G- $\Delta 9$ GL/DMGF virulence in swine}

ASFV-G- $\Delta 9$ GL virus has been shown to be attenuated when intramuscularly (IM) inoculated in swine at doses of $10^{2}$ or $10^{3} \mathrm{HAD}_{50}$ while animals inoculated with $10^{4} \mathrm{HAD}_{50}$ developed clinical disease similar to that observed in animals IM inoculated with $10^{4} \mathrm{HAD}_{50}$ of parental ASFV-G, the only difference being a slight delay in the onset of fever (O'Donnell et al., 2015b). In order to evaluate the additive effect of the deletion of MGF360/MGF505 genes to the ASFV-G- 49 GL genome, ASFV-G- $\Delta 9 \mathrm{GL} / \Delta \mathrm{MGF}$ was IM inoculated into three different groups ( $\mathrm{n}=5$ ) of $80-90$ pound pigs at doses of $10^{2}, 10^{4}$ or $10^{6} \mathrm{HAD}_{50}$ and compared with a group of animals inoculated with $10^{3}$ $\mathrm{HAD}_{50}$ of ASFV-G. As expected, animals infected with $10^{3} \mathrm{HAD}_{50}$ of ASFV-G exhibited increased 
body temperature ( $>104^{\circ} \mathrm{F}$ ) by 3 to 4 days post-infection followed by the appearance of clinical signs associated with the disease including anorexia, depression, purple skin discoloration, staggering gait and diarrhea (Table 1). Signs of the disease aggravated progressively over time and animals either died or were euthanized in extremis by around 7 days post-infection (pi). Animals infected with $10^{2}$, $10^{4}$ or $10^{6} \mathrm{HAD}_{50}$ of ASFV-G- $\Delta 9 \mathrm{GL} / \Delta \mathrm{MGF}$ did not present any signs of clinical disease associated with ASF during the entire observation period (21 days) (Table 1). Therefore, deletion of MGF36012L, 13L, 14L and MGF505-1R, 2R, 3R genes ( $\triangle$ MGF construct) significantly increased attenuation of ASFV-G- $\Delta 9 \mathrm{GL}$ since at doses that ASFV-G- $\Delta 9 \mathrm{GL}$ became virulent $\left(10^{4}\right.$ or $\left.10^{6} \mathrm{HAD}_{50}\right)$ (O'Donnell et al., 2015b), ASFV-G- $\Delta 9 \mathrm{GL} / \Delta \mathrm{MGF}$ is completely attenuated.

Viremia in experimentally inoculated animals was quantified at different days pi in swine macrophage cell cultures. As expected, animals inoculated with $10^{3} \mathrm{HAD}_{50}$ of virulent parental ASFV-G had very high virus titers in blood (as high as $10^{7}-10^{8} \mathrm{HAD}_{50} / \mathrm{ml}$ ) until the day of their death (Fig. 4). Conversely, animals inoculated with $10^{2}, 10^{4}$ or $10^{6} \mathrm{HAD}_{50}$ of mutant ASFV-G$\Delta 9 \mathrm{GL} / \Delta \mathrm{MGF}$ had either very low or undetectable (test sensitivity is $\geq 10^{1.8} \mathrm{HAD}_{50} / \mathrm{ml}$ ) virus titers in blood (Fig. 4). Therefore, although deletion of the MGF360/505 genes in the ASFV-G- $\Delta 9$ GL genome does not affect the ability of ASFV-G- $\Delta 9 \mathrm{GL} / \Delta \mathrm{MGF}$ to replicate in swine macrophage cultures, it certainly provokes a decreased replication during the infection in swine.

Presence and magnitude of viremias in animals infected with either ASFV-G- $\Delta 9$ GL or ASFV-G- $\triangle \mathrm{MGF}$ is quite heterogeneous, ranging from undetectable, particularly when low doses are used, to high titers $\left(10^{5}-10^{7} \mathrm{HAD}_{50} / \mathrm{ml}\right)$ (O’Donnell et al., 2015a, and 2015b). In addition, no correlation was established between level of viremia and presence of any ASFV-related clinical signs, since all inoculated animals remained clinically normal regardless of their viremia values. Interestingly, all ASFV-G- $\Delta 9 \mathrm{GL} / \Delta \mathrm{MGF}-$ infected animals presented almost undetectable viremias 
regardless if their virus yields in swine macrophage cultures were not drastically different from those of ASFV-G- $\triangle 9 \mathrm{GL}$ or ASFV-G- $\triangle \mathrm{MGF}$, suggesting that either our swine macrophage cultures are not an accurate representation of the macrophage population in vivo or, there are other unknown host immune factors besides the availability of cell types susceptible to infection that govern the kinetics of virus replication during the infection in the animal.

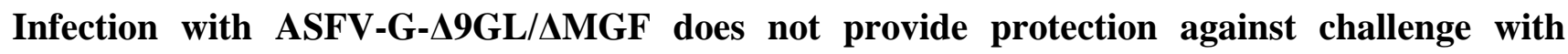 virulent ASFV-G}

In order to assess the effect of inoculation with ASFV-G- $\Delta 9 \mathrm{GL} / \Delta \mathrm{MGF}$ on the induction of protection against disease, ASFV-G- $\Delta 9 \mathrm{GL} / \Delta \mathrm{MGF}$ exposed animals were challenged with parental virulent ASFV-G. Animals previously infected IM with $10^{2}, 10^{4}$ or $10^{6} \mathrm{HAD}_{50}$ of ASFV-G$\triangle 9 \mathrm{GL} / \triangle \mathrm{MGF}$ were IM challenged at $28 \mathrm{dpi}$ with $10^{3} \mathrm{HAD}_{50}$ of virulent parental ASFV-G. Animals were monitored daily for clinical signs and changes in body temperature. Five naïve animals that were challenged with parental ASFV-G using the same route and dose served as a control group. These animals inoculated with ASFV-G displayed ASF-related signs by 3-4 days post-challenge $(\mathrm{dpc})$, evolving into a more severe disease in the following days and all animals dying or being euthanized around 6-7 dpc (Table 2). All animals receiving $10^{2}, 10^{4}$ or $10^{6} \mathrm{HAD}_{50}$ of ASFV-G$\Delta 9 \mathrm{GL} / \Delta \mathrm{MGF}$ succumbed to the challenge with the parental virulent virus. Kinetics of appearance of clinical signs, including rise of the body temperature, were very similar to those of the control animals challenged with ASFV-G and no significant differences were found in the length of the disease or time in which animals were euthanized between animals previously infected with ASFVG- $\Delta 9 \mathrm{GL} / \triangle \mathrm{MGF}$ and those just challenged with virulent parental ASFV-G (Table 2).

The presentation of ASF clinical signs, replication kinetics and magnitude of the viremia titers in the ASFV-G- $\triangle 9 \mathrm{GL} / \Delta \mathrm{MGF}-\mathrm{infected}$ animals after the challenge with virulent ASFV-G did not 
differ from that of the challenged control animals (Fig. 4). In general, absence of virus titers in blood upon challenge is a good indicator of the protective potency of the immunity elicited by a vaccine virus. In our experience, this is not always the case with ASFV and, particularly, with animals infected with ASFV-G- $\Delta 9$ GL or ASFV-G- $\Delta$ MGF which were protected against the disease/lethality produced by challenge with virulent ASFV-G (O'Donnell et al., 2015a, and 2015b). In the particular case of ASFV-G- $\triangle$ MGF-infected animals protected against the challenge, $30-40 \%$ of those showed presence of challenge virus in blood (O’Donnell et al., 2015a).

Presence of anti-ASFV antibodies in serum of animals inoculated with ASFV-G$\Delta 9 \mathrm{GL} / \Delta \mathrm{MGF}$ was detected at the time of challenge using an in-house developed direct ELISA (see Materials and Methods). While animals inoculated with ASFV-G- $\Delta$ 9GL or ASFV-G- $\Delta$ MGF presented at the time of challenge an average $(n=8)$ antibody titer of $\log _{10} 2.1(\mathrm{SD} \pm 0.32)$ and $\log _{10}$ $2.1(\mathrm{SD} \pm$ 0.57), respectively, none of the ASFV-G- $\Delta 9 \mathrm{GL} / \Delta \mathrm{MGF}-$ infected animals had detectable levels of antibodies at 28 days pi. As expected, induction of virus-specific antibodies is directly related to the presence of virus replication in the ASFV-G- $\Delta 9 \mathrm{GL} / \Delta \mathrm{MGF}-$ infected animals. In this regard, it should be mentioned that all ASFV-G- $\triangle 9$ GL- or ASFV-G- MGF-infected animals protected against disease after challenge with parental ASFV-G presented, at some point before challenge, significant viremia values, which was completely absent in all ASFV-G- $\Delta 9 \mathrm{GL} / \Delta \mathrm{MGF}-$ infected animals. Host mechanisms mediating protection against ASFV infection or disease still remain unknown and the possible relationship between replication of attenuated strains and the protection achieved by them cannot be ruled out at this time.

Therefore, infection with any of the doses of ASFV-G- $\Delta 9 \mathrm{GL} / \Delta \mathrm{MGF}$ tested equally failed in inducing protection against clinical disease, resulting in virus replication and death upon challenge with parental virulent ASFV-G. 
Genomic manipulation of ASFV as presented here has not been reported before; therefore, predictability of the obtained phenotype has some degree of uncertainty. Perhaps the only report providing comparable data was presented by Abrams et al., (2013) where it was shown that deletion of the NL (DP71L) and UK (DP96R) genes from the genome of the naturally attenuated OUR T88/3 strain reduced its ability to protect pigs against challenge with virulent virus. As reported here, deletion of virus genes previously associated with virus virulence (Zsak et al., 1996 and 1998) in the genome of an attenuated virus strain produced a deleterious effect in its ability to confer protection against challenge. 


\section{Conclusions}

The additive genetic effect on virus attenuation sought by deleting 9GL and MGF505/360 genes in ASFV-G led to a significant decrease of virus virulence in swine. ASFV-G- $\Delta 9 \mathrm{GL} / \mathrm{MGF}$ double-deletion mutant virus administered at doses 100-fold over the dose that causes disease in swine exposed to ASFV-G- $\triangle 9$ GL resulted in attenuation. However this particular combination of deletions in the ASFV-G genome led to a virus with limited immunogenic capability precluding the use of it as an effective modified live vaccine. Although pursuing additive variations for specific traits by genetically modifying ASFV is feasible as shown here, the outcome of those modifications is still unpredictable and at each instance it will require a thorough experimental corroboration. 


\section{Acknowledgements}

We thank the Plum Island Animal Disease Center Animal Care Unit staff for excellent technical assistance. We wish to particularly thank Mrs. Melanie V. Prarat for editing the manuscript. This project was funded through an interagency agreement with the Science and Technology Directorate of the U.S. Department of Homeland Security under Award Numbers HSHQDC-11-X00077 and HSHQPM-12-X-00005 and The State of Kansas National Bio and Agro-Defense Facility Fund (NBAF) and Institute for Infectious Animal Diseases (IIAD) Career Development Program. We would like to thank ARS/USDA-University of Connecticut SCA\# 58-1940-1-190 for partially supporting this work. 


\section{References}

Abrams, C. C., Goatley, L., Fishbourne, E., Chapman, D., Cooke, L., Oura, C.A., Netherton, C. L., Takamatsu, H. H., Dixon, L. K. 2013. Deletion of virulence associated genes from attenuated African swine fever virus isolate OUR T88/3 decreases its ability to protect against challenge with virulent virus. Virology 443:99-105.

Chapman, D.A., Darby, A.C., Da Silva, M., Upton, C., Radford, A.D., and Dixon, L.K., 2011. Genomic analysis of highly virulent Georgia 2007/1 isolate of African swine fever virus. Emerg Infec Diseas 17:599-605.

Coggins, L.1974. African swine fever virus. Pathogenesis. de la Vega, I., Viñuela, E., and Blasco, R. 1990. Genetic variation and multigene families in African swine fever virus. Virology 179:234246.

Costard, S., Porphyre, V., Messad, S., Rakotondrahanta, S., Vidon, H., Roger, F. and Pfeiffer, D.U. 2008. Exploratory multivariate analysis for differentiating husbandry practices relevant to disease risk for pig farmers in Madagascar. In Proc. 2008 Annu. Conf. of the Society for Veterinary Epidemiology and Preventive Medicine (SVEPM), Liverpool, England, pp. 228-238.

de la Vega, I., Viñuela, E., Blasco, R. 1990. Genetic variation and multigene families in African swine fever virus. Virology 179:234-46.Hamdy, F.M., and Dardiri, A.H. 1984 Clinic and immunologic responses of pigs to African swine virus isolated from the Western hemisphere. Am J Vet Res 45:711-714.

Kihm, U., Ackerman, M., Mueller, H., and Pool, R. 1987. Approaches to vaccination. In: African swine fever. Becker Y (ed) Martinus Nijhoff, Boston, pp 127-144.

Katz, D., Shi, W., Wildes. M. J., Krug, P. W., Hilliard, J. K. 2012. Reassessing the detection of Bvirus-specific serum antibodies. Comp Med 62: 516-526 
Krug, P.W., Holinka, L.G., O'Donnell, V., Reese, B., Sanford, B., Fernandez-Sainz, I., Gladue, D.P., Arzt, J., Rodriguez, L., Risatti, G.R., and Borca, M.V. 2015. The progressive adaptation of a georgian isolate of african Swine Fever virus to vero cells leads to a gradual attenuation of virulence in Swine corresponding to major modifications of the viral genome. J Virol 89:23242332.

Lewis, T., Zsak, L., Burrage, T.G., Lu, Z., Kutish, G.F., Neilan, J.G., and Rock, D.L., 2000. An African swine fever virus ERV1-ALR homologue, 9GL, affects virion maturation and viral growth in macrophages and viral virulence in swine. J Virol 74:1275-1285.

Mebus, C.A. 1988 African swine fever. Adv Virus Res 35:251-269.

Moore, D.M., Zsak, L., Neilan, J.G., Lu, Z., and Rock, D.L., 1998. The African swine fever virus thymidine kinase gene is required for efficient replication in swine macrophages and for virulence in swine. J Virol 72:10310-10315.

O'Donnell, V., Holinka, L.G., Gladue, D.P., Sanford, B., Krug, P.W., Lu, X., Arzt, J., Reese, B., Carrillo, C., Risatti, G.R., Borca, M.V. 2015a. African swine fever virus Georgia isolate harboring deletions of MGF360 and MGF505 genes is attenuated in swine and confers protection against challenge with virulent parental virus. J Virol 89:6048-56.

O'Donnell, V., Holinka, L.G., Krug, P.W., Gladue, D.P., Carlson, J., Sanford, B., Alfano, M. Kramer, E., Lu, X., Arzt, J., Reese, B., Carrillo, C., Risatti, G.R., Borca, M.V. 2015b. African swine fever virus Georgia 2007 with a deletion of virulence-associated gene 9GL (B119L), when administered at low doses, leads to virus attenuation in swine and induces an effective protection against homologous challenge. J Virol 89: 8556-66.

Pieres, S., Riveiro, G., and Costa, J.V. 1997. Sequence and organization of the left variable region 110 region of the Vero-adapted L60V strain of African swine fever virus. Virus Genes 15:271-274. 
Reed, L.J., and Muench, H.A., 1938. A simple method of estimating fifty per cent endpoints. Am J Hyg 27:493-497.

Ruiz Gonzalvo, F., Caballero, C., Martinez, J., and Carnero, M.E. 1986. Neutralization of African swine fever virus by sera from African swine fever-resistant pigs. Am J Vet Res, 47:1858-1862.

Tabares et al., 1987. Characterization of ASFV proteins. In: Becker, Y. (ed) African swine fever. Martinus Nijhoff, Boston, pp61-61.

Tulman, E.R., Delhon, G.A., Ku, B.K. and Rock, D.L. 2009 African Swine Fever Virus James L. Van Etten (ed.) Lesser Known Large dsDNA Viruses. 43 Current Topics in Microbiology and Immunology 328. Springer-Verlag Berlin Heidelberg.

Yanez, R.J., Rodriguez, J.M., Nogal, M.L., Yuste, L., Enriquez, L., Rodriguez, J.F., and Viñuela, E. 1995. Analysis of the complete nucleotide sequence of Africans swine fever virus. Virology 208:249-278.

Zsak, L., Caler, E., Lu, Z., Kutish, G.F., Neilan, J.G., and Rock, D.L. 1998. A nonessential African swine fever virus gene UK is a significant virulence determinant in domestic swine. J Virol 72:1028-1035.

Zsak, L., Lu, Z., Kutish, G.F., Neilan, J.G., and Rock, D.L. 1996. An African swine fever virus virulence-associated gene NL-S with similarity to the herpes simplex virus ICP34.5 gene. J Virol 70:8865-8871.

Zsak, L., Lu, Z., Burrage, T.G., Neilan, J.G., Kutish, G.F., Moore, D.M., and Rock, D.L. 2001. African swine fever virus multigene family 360 and 530 genes are novel macrophage host range determinants. J Virol 75:3066-3076. 
Table 1. Swine survival and fever response following infection with different doses of ASFV-G$\Delta 9 \mathrm{GL} / \Delta \mathrm{MGF}$.

\section{Fever}

\begin{tabular}{|c|c|c|c|c|c|}
\hline \multirow[b]{2}{*}{$\begin{array}{l}\text { Virus and Dose } \\
\text { (ASFV-G-) }\end{array}$} & \multirow[b]{2}{*}{$\begin{array}{c}\text { No. of } \\
\text { Survivors/total }\end{array}$} & \multirow[b]{2}{*}{$\begin{array}{l}\text { Mean time } \\
\text { to death } \\
\text { days }( \pm \mathrm{SD})\end{array}$} & \multicolumn{3}{|c|}{ Fever } \\
\hline & & & $\begin{array}{l}\text { No. of days } \\
\text { to onset } \\
( \pm \text { SD })\end{array}$ & $\begin{array}{c}\text { Duration, } \\
\text { No. of days } \\
\quad( \pm \text { SD })\end{array}$ & $\begin{array}{l}\text { Maximum } \\
\text { daily temp, } \\
{ }^{\circ} \mathrm{F}( \pm \mathrm{SD})\end{array}$ \\
\hline$\Delta 9 \mathrm{GL} / \Delta \mathrm{MGF} 10^{2} \mathrm{HAD}$ & $5 / 5$ & - & - & - & $102.9(0.79)$ \\
\hline$\Delta 9 \mathrm{GL} / \Delta \mathrm{MGF} 10^{4} \mathrm{HAD}$ & $5 / 5$ & - & - & - & $103.1(0.68)$ \\
\hline$\Delta 9 \mathrm{GL} / \Delta \mathrm{MGF} 10^{6} \mathrm{HAD}$ & $5 / 5$ & - & - & - & $102.7(0.47)$ \\
\hline Parental $10^{4} \mathrm{HAD}$ & $0 / 5$ & $7(0.71)$ & $3.6(0.55)$ & $3.4(1.1)$ & $105.9(0.62)$ \\
\hline
\end{tabular}

(-) Animals did not die or they did not present with fever. 
Table 2. Swine survival and fever response of ASFV-G- $\square$ GG/ $\square$ MGF-infected animals challenged with parental ASFV-G.

\section{Fever}

\begin{tabular}{|c|c|c|c|c|c|}
\hline \multirow[b]{2}{*}{$\begin{array}{l}\text { Virus and Dose } \\
\text { (ASFV-G-) }\end{array}$} & \multirow[b]{2}{*}{$\begin{array}{c}\text { No. of } \\
\text { Survivors/total }\end{array}$} & \multirow[b]{2}{*}{$\begin{array}{l}\text { Mean time } \\
\text { to death, } \\
\text { days }( \pm \mathrm{SD})\end{array}$} & \multicolumn{3}{|c|}{ Fever } \\
\hline & & & $\begin{array}{l}\text { No. of days } \\
\text { to onset } \\
\text { ( } \pm \text { SD) }\end{array}$ & $\begin{array}{c}\text { Duration, } \\
\text { No. of days } \\
( \pm \text { SD) }\end{array}$ & $\begin{array}{c}\text { Maximum } \\
\text { daily temp, } \\
{ }^{\circ} \mathbf{F}( \pm \mathrm{SD})\end{array}$ \\
\hline$\Delta 9 \mathrm{GL} / \Delta \mathrm{MGF} 10^{2} \mathrm{HAD}$ & $0 / 5$ & $7(0)$ & $4.4(1.14)$ & $2.6(1.14)$ & $105.5(1.69)$ \\
\hline$\Delta 9 \mathrm{GL} / \Delta \mathrm{MGF} 10^{4} \mathrm{HAD}$ & $0 / 5$ & $6.8(0.45)$ & $3.2(0.45)$ & $3.6(0.9)$ & $106.4(0.58)$ \\
\hline$\Delta 9 \mathrm{GL} / \Delta \mathrm{MGF} 10^{6} \mathrm{HAD}$ & $0 / 5$ & $7(0)$ & $3.2(0.45)$ & $3.8(0.54)$ & $106.1(0.45)$ \\
\hline Mock infected & $0 / 5$ & $6.6(0.55)$ & $3.8(0.45)$ & $2.8(0.84)$ & $105.7(0.55)$ \\
\hline
\end{tabular}

All animals were IM challenged with $10^{3} \mathrm{HAD}$ of parental ASFV-G. 


\section{Figure Legends}

Fig. 1. Schematic representation of the 9GL (B119L) and MGF360/MGF505 gene regions deleted in ASFV-G- $\Delta 9 \mathrm{GL} / \Delta \mathrm{MGF}$. Nucleotide positions indicating the boundaries of the deletion relative to the ASFV-G genome are indicated.

Fig. 2. PCR analysis of ASFV-G- $\Delta 9 \mathrm{GL} / \Delta \mathrm{MGF}$ stock using specific primers targeting MGF360/MGF505 or $\beta$-GUS genes. Viral DNA from ASFV-G- $\Delta 9$ GL/ $\Delta$ MGF or ASFV-G- $\Delta 9$ GL were tested for the presence of MGF360/MGF505 and $\beta$-GUS gene.

Fig. 3. In vitro growth kinetics of ASFV-G- $\Delta 9 \mathrm{GL} / \Delta \mathrm{MGF}$, ASFV-G- $\Delta 9 \mathrm{GL}$, ASFV-G- $\Delta \mathrm{MGF}$, and parental ASFV-G. Primary swine macrophage cell cultures were infected (MOI 0.01) with virus and virus yield, obtained at the indicated times pi, were titrated in primary swine macrophage cell cultures. Data represent means and standard deviations from two independent experiments. Sensitivity of virus detection: $\geq \log _{10} 1.8 \mathrm{HAD}_{50} / \mathrm{ml}$.

Fig. 4. Virus titers in blood samples obtained from pigs infected IM with either $10^{2}, 10^{4}$ or $10^{6}$ $\mathrm{HAD}_{50}$ of ASFV-G- $\triangle 9 \mathrm{GL} / \Delta \mathrm{MGF}$ and challenged at 28 days pi (arrow) with $10^{3} \mathrm{HAD}_{50}$ of ASFV-G. Control 1 and Control 2 represent animals inoculated with $10^{3} \mathrm{HAD}_{50}$ of ASFV-G at day 0 postinfection or challenge, respectively. Values are expressed as $\log _{10} \mathrm{HAD}_{50} / \mathrm{ml}$. Sensitivity of virus detection: $\geq \log _{10} 1.8 \mathrm{HAD}_{50} / \mathrm{ml}$. 
FIGURES

Fig. 1

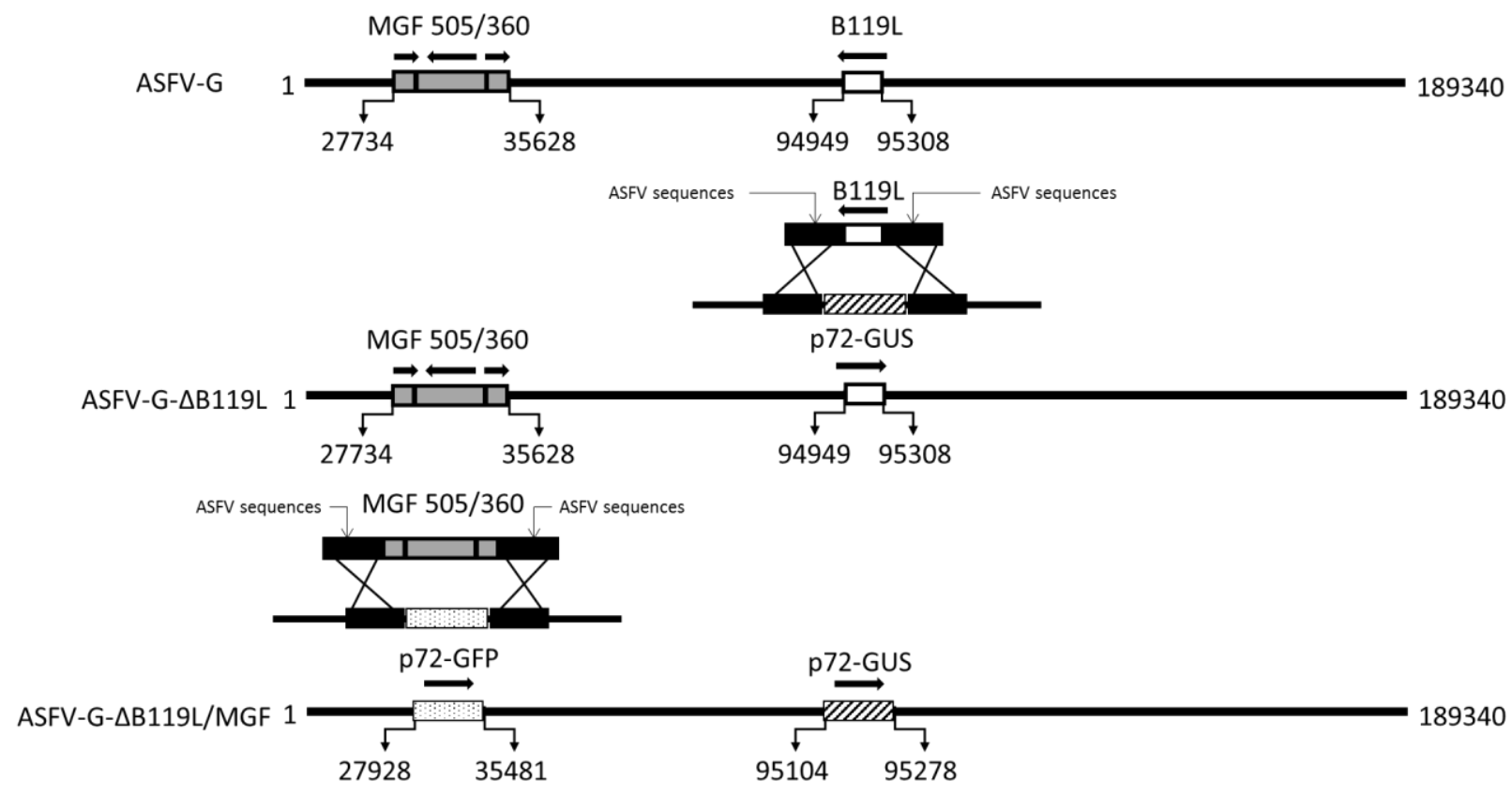


Fig. 2

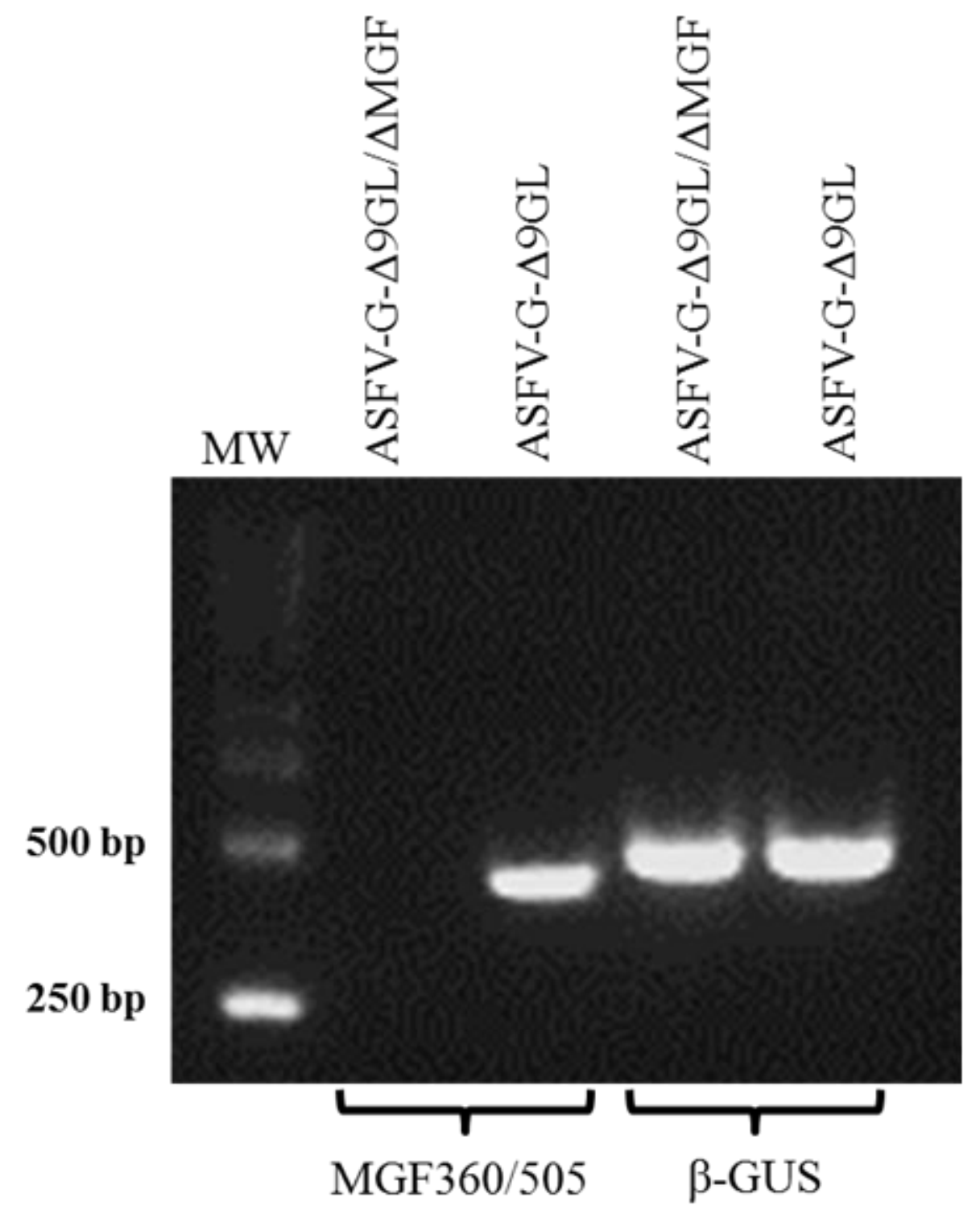


Fig. 3

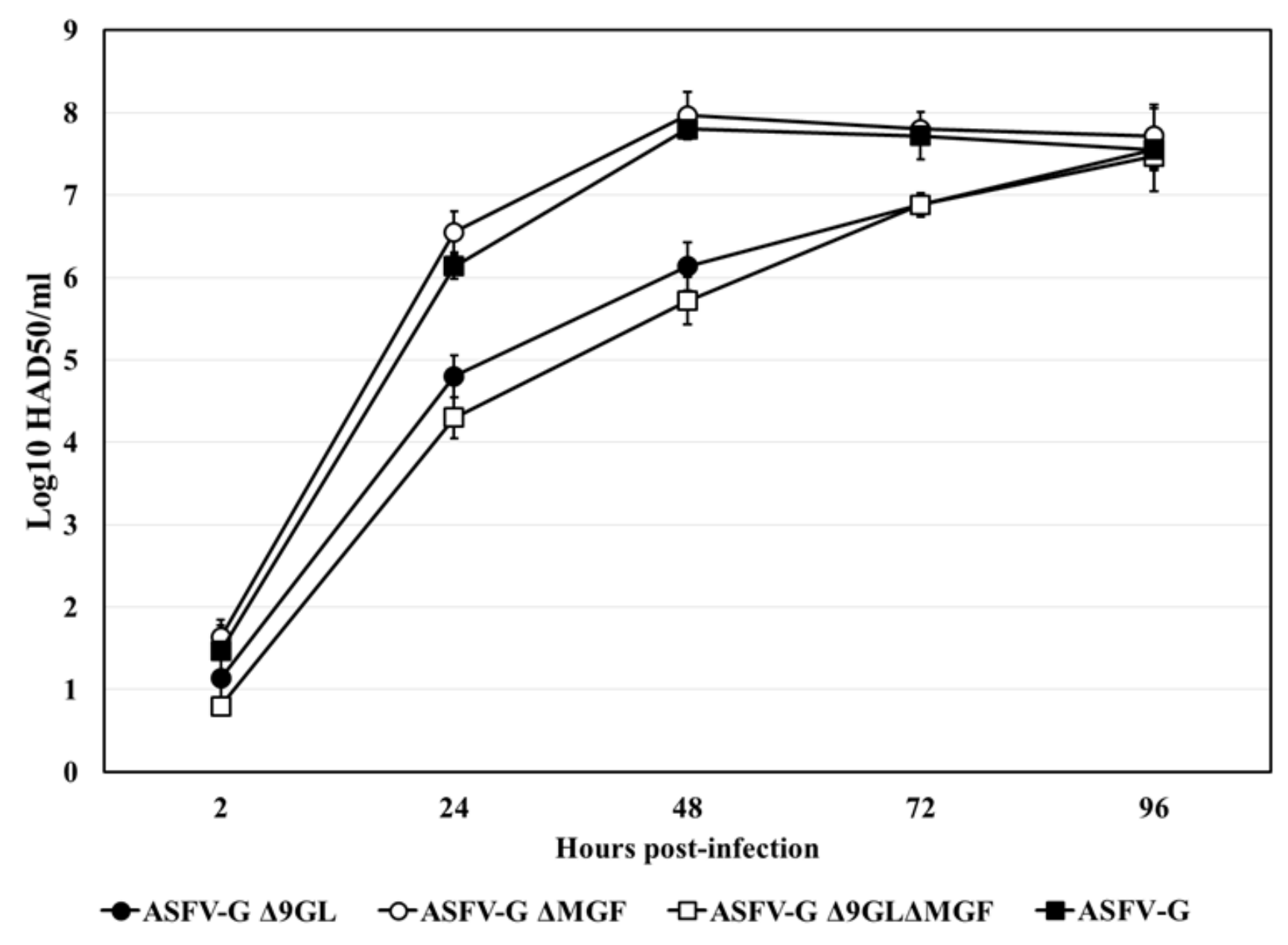


Fig. 4

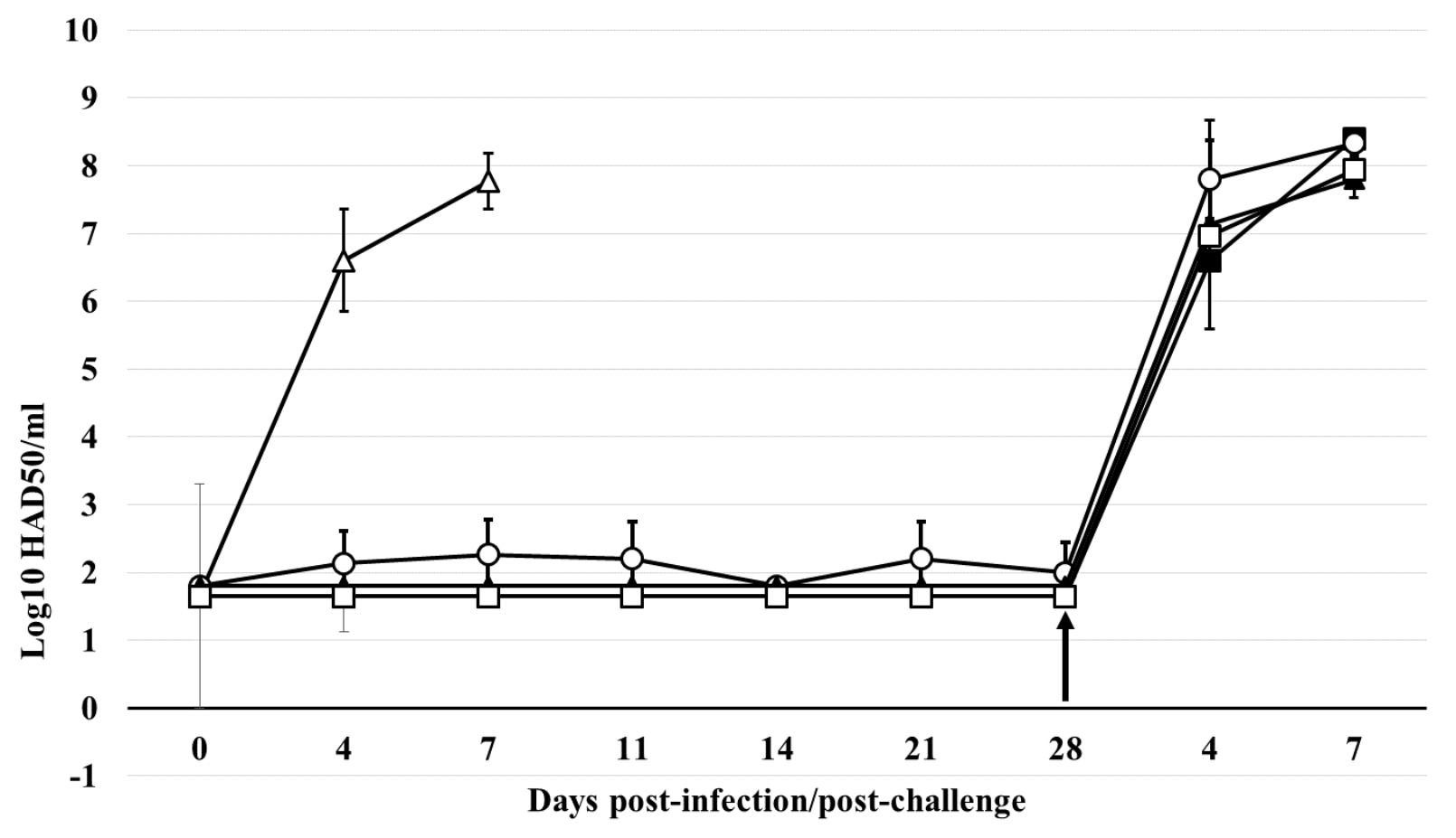

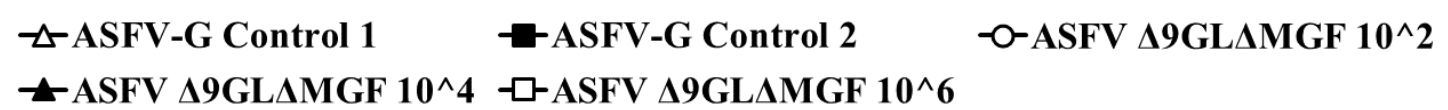

